\title{
NICE EFFICIENT PRESENTATIONS FOR ALL SMALL SIMPLE GROUPS AND THEIR COVERS
}

\author{
COLIN M. CAMPBELL, GEORGE HAVAS, COLIN RAMSAY AND \\ EDMUND F. ROBERTSON
}

\begin{abstract}
Prior to this paper, all small simple groups were known to be efficient, but the status of four of their covering groups was unknown. Nice, efficient presentations are provided in this paper for all of these groups, resolving the previously unknown cases. The authors' presentations are better than those that were previously available, in terms of both length and computational properties. In many cases, these presentations have minimal possible length. The results presented here are based on major amounts of computation. Substantial use is made of systems for computational group theory and, in particular, of computer implementations of coset enumeration. To assist in reducing the number of relators, theorems are provided to enable the amalgamation of power relations in certain presentations. The paper concludes with a selection of unsolved problems about efficient presentations for simple groups and their covers.
\end{abstract}

\section{Introduction}

For a finite group $G$, the group $H$ is a stem extension of $G$ if there is $A \leqslant Z(H) \cap H^{\prime}$ with $G \cong H / A$. A stem extension of maximal order is called a covering group of $G$ and the maximal $A$ in this case is the Schur multiplier of $G$, denoted by $M(G)$. If $G$ is perfect, then $G$ has a unique covering group, which we denote by $\widehat{G}$. The deficiency of a finite presentation $\{X \mid R\}$ of $G$ is $|R|-|X|$. The deficiency of $G, \operatorname{def}(G)$, is the minimum of the deficiencies of all finite presentations of $G$. For a good overview of Schur multipliers and related topics, see [24], where Corollary 1.2 shows that $\operatorname{rank}(M(G))$ is a lower bound for $\operatorname{def}(G)$. The group $G$ is said to be efficient when this lower bound is achieved.

Deciding whether a given group is efficient may be difficult; indeed, the problem is unsolvable in general [1]. Previous work has used a variety of techniques to try to find efficient presentations. In particular, considerable effort has been put into showing that simple groups and their covering groups are efficient. We give the names of simple groups in Atlas format [10]. A survey of results for simple groups of order up to one million has been given by Campbell, Robertson and Williams [8]. Subsequent to this, $L_{3}(5)$ has been shown to be efficient by Campbell, Havas, Hulpke and Robertson [7], and $\widehat{M}_{22}$, the covering group of the Mathieu group $M_{22}$, has been shown to be efficient by Havas and Ramsay [18].

No comprehensive, systematic approach to finding such efficient presentations has previously been taken. One basic method has been to start with a presentation on a minimal

The second and third authors were partially supported by the Australian Research Council, the Edinburgh Mathematical Society and the UK Network in Computer Algebra.

Received 3 October 2003, revised 16 September 2004; published 18 October 2004.

2000 Mathematics Subject Classification 20-04, 20D05, 20D06, 20D08, 20 F05.

(C) 2004, Colin M. Campbell, George Havas, Colin Ramsay and Edmund F. Robertson 
generating pair for these groups (see [5] for the definition), and then to try combining relators. Intuition has suggested that presentations on a pair of generators will be more likely to lead to presentations that could be reduced to efficient ones than those on larger generating sets. When this approach has failed, other ad hoc methods have been used.

We report on systematic attempts to look for efficient presentations of small simple groups and their covering groups. By 'small', we mean that the underlying simple group has order at most $10^{5}$. One systematic approach examines presentations on all distinct generating pairs for the groups in a search for short efficient presentations and for efficient presentations that lead to easy coset enumerations. A second approach involves looking at short presentations of perfect groups. A third approach generates special kinds of relators.

In the process of finding 'nice' presentations, we resolve all previously unresolved problems about the efficiency of small simple groups and their covers: we show that $\widehat{L}_{3}(4)$, $\widehat{A}_{8}, \widehat{S}_{4}(3)$ and $\widehat{M}_{12}$ are efficient by giving efficient presentations for them. To facilitate the construction of efficient presentations, we give theorems that show how to combine certain kinds of power relations in presentations for simple groups and their stem extensions so that we obtain presentations for covering groups.

There is an undefined term in the title: 'nice'. What makes an efficient presentation a nice one? One view is that short presentations are nicer than longer ones. Also, since presentations may be used as input into group-theoretic programs, we want them to behave well in that role. An important computer procedure for finitely presented groups is coset enumeration, and we assess the presentations in terms of their behaviour as targets of coset enumeration. Further, in view of our theorems for combining power relations, presentations that include relators showing the order of generators are nice. We list instances of nice efficient presentations found by our methods.

Since the groups $L_{2}(p)$ for primes $p \geqslant 5$ have been covered by Sunday [23], and $\widehat{L}_{2}(p)$ by Campbell and Robertson [3], we consider the other small simple groups here. This is not meant to imply that the general presentations given in those papers provide the shortest presentations for the individual linear groups and their covering groups, but merely explains why we omit them from this paper.

\section{Methodology}

The availability of systems for computational group theory (for example, GAP [14], MAGMA [2] and Magnus [21]) makes it quite easy to experiment with groups. Havas, Newman and O'Brien [19] have developed a MAGMa program that enables us to find all distinct generating sets for moderately sized permutation groups. (The program uses representatives from appropriately merged orbits of the action of the automorphism group of each permutation group studied.) Our first method is to use this program to find such distinct generating pairs for groups under consideration, and then to use the built-in algorithm of MAGMA to find a presentation of the group on each of these generating sets.

Presentations found in this way tend to have a reasonably low number of relators, but are rarely efficient, even for small groups. However, often simply checking all efficient-sized subsets of the relators reveals efficient presentations. These checks are done by first quickly checking that a subset presents a perfect group (otherwise, it does not present a group that we are seeking). Note that here we might be looking for either the underlying simple group, or some stem extension. If this test is passed, we attempt to check that the presentation is correct by coset enumeration. We use the ACE enumerator [17], either as available in GAP or MAGMA, or as a stand-alone program for some more difficult cases. 
Most of our groups succumb to this straightforward search. For those that do not, we attempt to amalgamate relations, again preserving the property that the amalgamated presentation is that of a perfect group. Here we start with small, but not efficient, sets of relators. In particular, we give in Section 4 one theorem that shows how to combine certain kinds of power relations so that we obtain presentations for covering groups.

Our second source of nice presentations for small simple groups and their covers is provided by censuses of short presentations of perfect groups, extending work by Havas and Ramsay [18]. The extension includes: two-generator, two-relator presentations with length up to 24; two-generator, three-relator presentations with length up to 26 ; three-generator, three-relator presentations with length up to 20; and one-relator quotients of $C_{l} * C_{m}$ (the free product of an $l$-cycle and an $m$-cycle) for coprime $l$ and $m$. (By 'a one-relator quotient of a particular group', we mean a presentation obtained by adding one extra relator to a presentation for the specified group.)

The third method extends the idea of enumerating one-relator quotients of $C_{l} * C_{m}$ to building appropriate one-relator quotients that present a simple group - or a stem extension - by computing and testing relators that hold. This is an easy modification of the process described by Campbell, Havas, Hulpke and Robertson [7] using the GAP program PGRelFind (see $[13,15])$.

When we find a suitable one-relator quotient of $C_{l} * C_{m}$, Theorem 4.1 enables us to build efficient presentations for the cover of the underlying simple group, and for all quotients of that cover.

One problem that we face in producing our table of new presentations is that once we are able to find an efficient presentation for a group, then there are arbitrarily many. In Table 2, we give an instance of a presentation with the shortest-found length (which is often the shortest possible length on two generators). It is chosen such that, within the presentations for the group of that length that we have investigated, it has best cosetenumeration performance. We measure coset-enumeration performance by the total number of cosets used in a successful enumeration of the trivial subgroup using the Hard strategy of ACE, with the group generators given in alphabetical order.

We take the presentation as produced by our process and generally do not make efforts like those described in [16] to improve it. Thus we give presentations produced by MAGMA without modification, including various relators in what may seem less natural forms (in the sense that inversion or cyclic rotation may produce more usual forms). In contrast, presentations from censuses of short presentations arise with relators in canonic form, as described in [18]. Generally speaking, there are often longer presentations that enumerate better. We comment on each of the groups in Section 5.

As far as the reliability of our results is concerned, we claim that all the presentations given in this paper correctly define the groups. Each presentation that appears has been verified by both GAP and MAGMA programs to present the specified group.

\section{Results}

In this section we give nice, efficient presentations for simple groups of order less than $10^{5}$ and for their covering groups, excluding $L_{2}(p)$ for $p$ prime. For convenience, we adopt the convention of using upper-case letters to denote inverses in presentations so that, for example, $A=a^{-1}$. We denote the commutator $A B a b$ by $[a, b]$. We give presentations that list sequences of relators and/or relations. For coset-enumeration purposes, the generators are always given in alphabetical order. 
Table 1: Previous shortest efficient presentations

\begin{tabular}{|c|c|c|c|c|}
\hline Name & Relators and/or relations & Length & Cosets & Ref. \\
\hline$A_{6}$ & $a^{2}=(a b)^{5}, b^{4},\left(a b^{2}\right)^{5}$ & 29 & 2609 & {$[5]$} \\
\hline$\widehat{A}_{6}$ & $a b^{3}(b a)^{-4},\left(a b^{2} a b^{-2}\right)^{2} a b^{2}$ & 27 & 16278 & {$[22]$} \\
\hline$L_{2}(8)$ & $x y X y x Y, x^{4}\left(y^{2} x y\right)^{2} y$ & 19 & 855 & {$[4]$} \\
\hline$A_{7}$ & $a^{3}=\left(a b^{2}\right)^{4}, b^{5},\left(b^{2} A b a\right)^{2}$ & 28 & 4144 & {$[4]$} \\
\hline$\widehat{A}_{7}$ & $a^{3}=\left(a b^{2}\right)^{4}, b^{5}=\left(b^{2} A b a\right)^{2}$ & 24 & 111022 & [4] \\
\hline$L_{2}(16)$ & $x y X y x Y, x y x^{4} y x y^{3} x^{3} y^{3}$ & 23 & 7852 & {$[4]$} \\
\hline$L_{3}(3)$ & $a^{2} B^{3}, B A(b a)^{5}(B A)^{7}(b A)^{3}(B a)^{2} b a(B a)^{2}(b a)^{2}$ & 51 & 148825 & {$[5]$} \\
\hline$U_{3}(3)$ & $B^{2} A B a^{3} B A, b^{2} A B^{2} A b^{2} a B a$ & 20 & 26722 & {$[19]$} \\
\hline$L_{2}(25)$ & $a^{2}, b^{3},(a b)^{2}(A B)^{2}(a b)^{2}(A B)^{4}(a b)^{5}(A B)^{4}$ & 43 & 8828 & {$[4]$} \\
\hline$\widehat{L}_{2}(25)$ & $x y X y x Y, x^{2} y^{2} x^{2} y^{4} x^{5} y^{4}$ & 25 & 22397 & [4] \\
\hline$M_{11}$ & $a b a^{4} b^{3}, b a b A B a B a b A$ & 19 & 13822 & [20] \\
\hline$L_{2}(27)$ & $a^{2}, b^{3},(a b)(A B)^{2}(a b)^{3}(A B)^{2}(a b)(A B)^{8}$ & 39 & 11269 & [4] \\
\hline$\widehat{L}_{2}(27)$ & $x y X y x Y, x\left(y x^{5} y\right)^{2} x y^{7}$ & 29 & 1759965 & {$[4]$} \\
\hline \multirow[t]{2}{*}{$A_{8}$} & $a^{2} B^{4},(a b)^{15}\left(a b^{2}\right)^{4}$ & & & \\
\hline & $(a b)^{6} b a b(a B)^{2}(a b)^{2} a B(a b)^{7} a B$ & 89 & 636449 & {$[5]$} \\
\hline$\widehat{A}_{8}$ & not known to be efficient & & & \\
\hline \multirow[t]{2}{*}{$L_{3}(4)$} & $a^{2}=(a b)^{7},\left(a b^{2}\right)^{5}=b^{4},\left(b(a b)^{3}\right)^{7}$ & & & \\
\hline & $b(a b)^{3} b(b a)^{4} b^{2}(a b)^{3}(b a)^{3} b^{2} a B(a b)^{3}(b a)^{3} b^{2} a b$ & 128 & 288596 & {$[5]$} \\
\hline$\widehat{L}_{3}(4)$ & not known to be efficient & & & \\
\hline \multirow[t]{2}{*}{$S_{4}(3)$} & $a^{2}=(a b)^{9},[a, b]^{4}=b^{4}$ & & & \\
\hline & $(a b)^{2} b a B(a b)^{3} a B\left(a b^{2} a b\right)^{3} b(a B)^{3}$ & 73 & 13658840 & [5] \\
\hline$\widehat{S}_{4}(3)$ & not known to be efficient & & & \\
\hline \multirow[t]{2}{*}{$\mathrm{Sz}(8)$} & $a^{2}=(a b)^{7},\left(a b^{2}\right)^{13},[a, b]^{13}=b^{4}$ & & & \\
\hline & $a b\left(a b^{2}(a B)^{2}\right)^{2} a b a B\left(a b^{2}\right)^{6}$ & 145 & 2927643 & [5] \\
\hline$\widehat{\operatorname{Sz}}(8)$ & $a^{2}=(a b)^{5},\left(a b^{2} a B\right)^{2}\left(a B^{2} a b\right)^{2} a=(b a B a)^{5} b^{2}$ & 53 & 227854383 & {$[4]$} \\
\hline$L_{2}(32)$ & $x y X y x Y,(x y x)^{2} y^{3} x^{5} y^{3}$ & 23 & 37177 & {$[4]$} \\
\hline$L_{2}(49)$ & $a^{2}, b^{3},(a b)(A B)^{2}\left((a b)^{2}(A B)^{3}(a b)^{2}\right)^{2}(A B)^{2}$ & 45 & 60069 & [4] \\
\hline$\widehat{L}_{2}(49)$ & $x y X y x Y, x y^{2}\left(x^{2} y^{3} x^{2}\right)^{2} y^{2}$ & 25 & 141922 & {$[4]$} \\
\hline$U_{3}(4)$ & $a^{2}=b^{3}, a^{3} b(a B)^{2}(a b)^{2} a B a b(a B)^{4} a b a B(B A)^{8} b$ & 50 & 602000 & {$[5]$} \\
\hline$M_{12}$ & $a^{2}=b^{3},(a b)^{10}=[a, b]^{6},\left((a b)^{4} a B a b a B\right)^{3}$ & 87 & 243246 & {$[5]$} \\
\hline$\widehat{M}_{12}$ & not known to be efficient & & & \\
\hline
\end{tabular}


We put our results in context by comparing them with the previous shortest efficient presentations explicitly published, and we provide citations for those earlier presentations (see Table 1). Sometimes those presentations imply the existence of shorter presentations. For example, where presentations include relators like $a^{2}$ and $b^{3}$, or like $a^{2} b^{3}$, it is possible to follow the ideas explained in [4] to give shorter presentations on $x=a b$ and $y=a B$ or $y=A B$.

The tables give the name of the simple group, relators and/or relations for the group, the total length of the relators in the corresponding presentation (freely and cyclically reducing relators as done by $\mathrm{ACE}$ ), and the total number of cosets used in a successful coset enumeration for this presentation over the trivial subgroup using the Hard strategy of the ACE enumerator. In Table 1 we provide a citation, and in Table 2 we provide a reference to the subsection in which we give further information on the group.

Table 2: Our short presentations

\begin{tabular}{|c|c|c|c|c|c|}
\hline Name & Relators & Length & Cosets & Order & Ref. \\
\hline$A_{6}$ & $a^{4}, b^{5}, a b a B a b a B A B$ & 19 & 1546 & 360 & 5.1 \\
\hline$\widehat{A}_{6}$ & $a^{3} b A^{2} b, a b^{2} A b A B^{3} a B$ & 18 & 2210 & 2160 & 5.1 \\
\hline$L_{2}(8)$ & $a^{2} b A B A b, a b A b a b^{2} A B^{2} a B$ & 19 & 592 & 504 & 5.2 \\
\hline$A_{7}$ & $a^{5}, a b a b a B^{3},\left(a^{2} b A B\right)^{2}$ & 23 & 3253 & 2520 & 5.3 \\
\hline$\widehat{A}_{7}$ & $a^{4} b A b A b, a^{2} b a b^{2} A^{2} B^{2}$ & 19 & 43805 & 15120 & 5.3 \\
\hline$L_{2}(16)$ & $a^{3} b^{2} A^{2} b^{2}, a b A B^{2} A b a B$ & 18 & 21825 & 4080 & 5.4 \\
\hline$L_{3}(3)$ & $a^{3} b A b^{2} A B, a^{2} b^{2} a B^{2} A b A b^{2}$ & 21 & 158892 & 5616 & 5.5 \\
\hline$U_{3}(3)$ & $a^{3} b A b A b, a^{2} b^{2} A B^{3} A b^{2}$ & 19 & 198076 & 6048 & 5.6 \\
\hline$L_{2}(25)$ & $a^{5}, a^{2} b^{2} a^{2} b^{2}, a b^{3} A b^{3} a B$ & 23 & 14917 & 7800 & 5.7 \\
\hline$\widehat{L}_{2}(25)$ & $a^{2} b A B A B A b, a^{3} B a b^{3} a B$ & 19 & 16779 & 15600 & 5.7 \\
\hline$M_{11}$ & $b A^{3} b A b^{3}, b a B A B A b a B a$ & 19 & 10428 & 7920 & 5.8 \\
\hline$L_{2}(27)$ & $(a b)^{2}, a^{7}, a^{2} b A b a B A b^{4}$ & 23 & 10509 & 9828 & 5.9 \\
\hline$\widehat{L}_{2}(27)$ & $a^{6} b A b, a^{3} B A b^{4} a B^{2}$ & 21 & 47253 & 19656 & 5.9 \\
\hline$A_{8}$ & $\left(a^{2} b\right)^{2}, a^{7}, a b A B^{3} A b A B^{2}$ & 24 & 427065 & 20160 & 5.10 \\
\hline$\widehat{A}_{8}$ & $a^{5} b A^{2} b, a^{2} b^{2} A B a b^{2} A B a B$ & 22 & 1252222 & 40320 & 5.10 \\
\hline$L_{3}(4)$ & $a^{5}, b^{5},(a b)^{3}, a^{2} B^{2} A B a B^{2} a b A B$ & 29 & 30500 & 20160 & 5.11 \\
\hline$\widehat{L}_{3}(4)$ & $a^{4} b A b A b, a^{2} b a b^{2} a B^{2} A B A^{2} b$ & 23 & 30181644 & 967680 & 5.11 \\
\hline$S_{4}(3)$ & $a^{5}, a b^{2} a b^{2}, a^{2} B a B a b a B^{2}$ & 21 & 26561 & 25920 & 5.12 \\
\hline$\widehat{S}_{4}(3)$ & $a^{5} b^{4}, a b A b A b A B^{2} a B^{2}$ & 21 & 166020 & 51840 & 5.12 \\
\hline $\mathrm{Sz}(8)$ & $B^{5}, A^{7}, A B^{2} a^{3} b a^{2} B, B a B A B A^{2} b^{2} a^{2} b a$ & 35 & 29420 & 29120 & 5.13 \\
\hline$\widehat{\mathrm{Sz}}(8)$ & $a^{3} b A^{2} b, a b^{2} a b^{2} a b^{6} a B$ & 22 & 346104 & 116480 & 5.13 \\
\hline$L_{2}(32)$ & $a^{2} b A B A b, a b A b A b A b a b^{5}$ & 21 & 35631 & 32736 & 5.14 \\
\hline$L_{2}(49)$ & $a^{4}, b^{5}, a^{2} B a^{2} B a b a b a B$ & 21 & 223362 & 58800 & 5.15 \\
\hline$\widehat{L}_{2}(49)$ & $B A B^{2} A b^{2} A B, A^{2} B a^{3} B A^{2} b$ & 19 & 214508 & 117600 & 5.15 \\
\hline$U_{3}(4)$ & $a^{4} b A b A b, a^{3} B A b^{2} A B A^{2} B^{2}$ & 22 & 1557671 & 62400 & 5.16 \\
\hline$M_{12}$ & $(B a)^{3}, a^{5} b^{6}, a b^{2} a B a^{2} b a^{2} b^{2}$ & 29 & 119334 & 95040 & 5.17 \\
\hline$\widehat{M}_{12}$ & $x y x Y X Y, x^{2} Y^{3} x^{2} Y x Y x^{2} Y^{2} x Y x Y^{5} x^{5}$ & 33 & 11717995 & 190080 & 5.17 \\
\hline
\end{tabular}


In earlier work on presentations for simple groups, Đoković [12] pointed out the difficulty of finding shortest presentations in general. His length measure is somewhat more complicated than ours, but his sentiments still apply.

We mention that the problem of finding a shortest presentation for a given finite group $G$ is quite open. We do not know the answer even in the case when $G$ is a cyclic group of prime order.

Our census-based approach enables us to provide shortest presentations on fixed numbers of generators for many of the groups under consideration here. The proof is obtained by adequately identifying all shorter possible presentations, extending the ideas in [18]. Indeed, all the presentations in Table 2 with length less than 20 are the shortest possible on two generators.

\section{An amalgamation theorem}

Group presentations frequently include relators that specify the orders of the group generators. When we have suitable relators like this in a presentation for a simple group (or a stem extension), we can effectively amalgamate them and obtain a presentation for a stem extension of the original simple group. We start with an amalgamation theorem that handles the situation where we have three relators: two giving generator orders, plus one other relator.

THEOREM 4.1. Let $G$ be a finite simple group. Suppose that $G$, or some stem extension of $G$, can be presented as

$$
P=\left\{a, b \mid a^{p}=b^{q}=w(a, b)=1\right\} .
$$

Then the covering group of $G$, all stem extensions of $G$, and $G$ itself, are efficient.

Proof. If we show that the covering group of $G$ is efficient, then the efficiency of $G$, and all stem extensions of $G$, follows by adding relations to kill factors in $M(G)$.

Let $e_{a}$ and $e_{b}$ be the exponent sums of $a$ and $b$ in $w(a, b)$ respectively. Note that $\left(e_{a}, p\right)=$ $\left(e_{b}, q\right)=1$ since, by assumption, the group presented by $P$ is perfect.

Assume first that $p e_{b}+q e_{a}=1$. Then consider the group $H$ with presentation

$$
\left\{a, b \mid a^{p} b^{-q}=w(a, b)=1\right\} .
$$

Now $H$ is a stem extension of $\langle P\rangle$ and, by assumption, $\langle P\rangle$ is a stem extension of the simple group $G$. Let $A=H, B=H$, and take $C$ to be the second centre of $H$. Since [ $B, C], A]=$ 1 and $[[C, A], B]=1$, then, by the Three Subgroup Lemma, $[[A, B], C]=1$. But $H$ is perfect, so $C=Z(H)$. Now $H$ is perfect, $H / Z(H) \cong G$, and $H$ has trivial multiplier (since it has a balanced presentation), so it follows that $H$ is the covering group of $G$.

Now consider the case where $p e_{b}+q e_{a} \neq 1$. Certainly, $(p, q)=1$ since $\langle P\rangle$ is perfect, so there exist $m, n$ with $p m+q n=1$. Choose $s$ and $t$ so that $s e_{a} \equiv 1 \bmod p$ and $t e_{b} \equiv 1 \bmod q$, and consider the transformation $a \longrightarrow a^{s n}, b \longrightarrow b^{t m}$. Then $w \longrightarrow \widetilde{w}$ with $a$-exponent sum congruent to $n \bmod p$ and $b$-exponent sum congruent to $m \bmod q$ so, inserting powers of $a^{p}$ and $b^{q}$ into $\widetilde{w}$ if necessary, we can transform $P$ to $\widetilde{P}$ where

$$
\widetilde{P}=\left\{a, b \mid a^{p}=b^{q}=\widetilde{w}(a, b)=1\right\} .
$$

Now $\langle P\rangle=\langle\widetilde{P}\rangle$, but $\widetilde{w}$ has the property that it satisfies $e_{a}=n$ and $e_{b}=m$, and so in $\widetilde{P}$ we have $p e_{b}+q e_{a}=1$, as required. 
Theorem 4.1 has already been applied by Havas, Newman and O'Brien [19] to obtain special kinds of presentations for $U_{3}(3)$.

Corollary 4.2. Let $G$ be a finite simple group. Suppose that $G$, or some stem extension of $G$, can be presented as

$$
P=\left\{a, b \mid u(a, b)^{p}=v(a, b)^{q}=w(a, b)=1\right\} .
$$

Suppose also that $u(a, b)$ and $v(a, b)$ generate the free group on $a$ and $b$. Then the covering group of $G$, all stem extensions of $G$, and $G$ itself, are efficient.

Proof. Let $r=u(a, b)$ and $s=v(a, b)$. Then $G$ can be presented as

$$
\left\{a, b, r, s \mid u(a, b)^{p}=v(a, b)^{q}=w(a, b)=1, r=u(a, b), s=v(a, b)\right\} .
$$

However, since $u(a, b)$ and $v(a, b)$ generate the free group on $a$ and $b$, we can write $a=U(r, s)$ and $b=V(r, s)$. Add these relations to the presentation for $G$, and then use them to eliminate $a$ and $b$. The relations $r=u(a, b)$ and $s=v(a, b)$ vanish when we substitute $a=U(r, s)$ and $b=V(r, s)$, since $u(a, b)$ and $v(a, b)$ are free generators.

We thus have a presentation for $G$ of the form

$$
P=\left\{r, s \mid r^{p}=s^{q}=W(r, s)=1\right\},
$$

and we can apply Theorem 4.1 .

A natural extension of Theorem 4.1 gives methods for amalgamating relations, given a presentation for (a stem extension of) a simple group with more relations, such as

$$
P=\left\{a, b \mid a^{p}=b^{q}=w_{1}(a, b)=\ldots=w_{n}(a, b)=1\right\} .
$$

We point out that our primary focus is on presentations that are efficient in terms of deficiency. This does not always coincide with best presentations for other purposes. In particular, for deficiency-zero groups, the deficiency-one presentation

$$
\left\{a, b \mid a^{p}=b^{q}=w(a, b)=1\right\}
$$

is likely to be much more useful for practical computation than the efficient presentation produced by Theorem 4.1,

$$
\left\{a, b \mid a^{p} b^{-q}=\widetilde{w}(a, b)=1\right\} .
$$

For example, these deficiency-one presentations are better for coset enumeration than the corresponding efficient presentations. Likewise, a presentation explicitly involving an involutory generator ( $a$, say, with the presentation including the relator $a^{2}$ ) is generally better for coset enumeration than presentations without such a relator, because coset-enumeration programs usually save space and time by utilizing the fact that $a=a^{-1}$. In our commentary on each group in the next section, we provide some information on such presentations.

\section{Commentary}

Our methodology produces a very large number of efficient presentations for most of the groups under consideration. Then simple modifications to these lead to many more presentations that are efficient. In our tables we have given the shortest efficient presentation that arose as one of our generated presentations, and that enumerates with the least total 
cosets over the trivial subgroup. We emphasise that we use this purely as a measure of cosetenumeration performance; we do not suggest that enumerations over the trivial subgroup are the best way to compute with the presentation to gain other information about the group. We also give further information for each simple group.

\section{1. $A_{6}$}

For $A_{6}$, we investigated thirty-three distinct generating pairs, and found eight efficient presentations amongst presentations on these generating sets. Four of these were one-relator quotients of $C_{4} * C_{5}$ with length 19 , like the tabulated presentation which arises in both a census of one-relator quotients of $C_{4} * C_{5}$ and in a census of short two-generator, threerelator presentations. Two other presentations on the distinct generating pairs were onerelator quotients of $C_{2} * C_{5}$. A shortest instance of one of these,

$$
\left\{a, b \mid(a b)^{2}, a^{5}, a b^{2} A b A b^{2} a B^{2}\right\}
$$

(one longer than the tabulated presentation), uses a total of 501 cosets, which is much better than our tabulated presentation.

We can use Theorem 4.1 to construct an efficient presentation for $\widehat{A}_{6}$ from any of the one-relator quotients of $C_{l} * C_{m}$. We also find forty-nine efficient presentations from the 978 distinct generating pairs of the group. We tabulate a presentation from a census of short two-relator presentations for perfect groups. It is one of two length-18 canonic presentations for the group, and can be obtained by amalgamating the power relations in a presentation for $A_{6}$ which is a one-relator quotient of $C_{2} * C_{5}$. It follows from the analysis of shorter presentations in [18] that this is a shortest possible two-relator presentation for $\widehat{A}_{6}$.

The best efficient canonic presentation for $A_{6}$ with respect to total cosets that we have found is

$$
\left\{a, b \mid a^{4}, a b^{2} a B^{3}, a b a B A B a B A B\right\},
$$

which uses a total of 392 cosets. Note that the deficiency-two presentation

$$
\left\{a, b \mid(a b)^{2}, a^{5}, b^{5},(a B)^{4}\right\}
$$

(already investigated as $(5,5 \mid 2,4)$ by Coxeter [11]) allows enumeration of the cosets of the trivial subgroup using a total of 360 cosets - that is, without the definition of any redundant cosets.

\section{2. $L_{2}(8)$}

For $L_{2}(8)$, we investigated eighty-five distinct generating pairs, but did not find any efficient presentations directly. However, among the three-relator subsets of presentations we found fifteen one-relator quotients of $C_{2} * C_{9}$, thirteen of $C_{2} * C_{7}$ and one of $C_{2} * C_{3}$, which present the group. Presentations for $L_{2}(8)$ also arise as one-relator quotients of $C_{3} * C_{7}$. Theorem 4.1 enables us to construct an efficient presentation for $L_{2}(8)$ from any of these. We tabulate a presentation from a census of short two-relator presentations for perfect groups. The shortest such presentations that we have found have length 19, and we found sixteen different canonic ones. The group arises implicitly as a one-relator quotient of $C_{2} * C_{3}$ via one of the length-19 canonic presentations:

$$
\left\{x, y \mid x y x Y X Y, x^{4} Y^{2} x Y^{3} x Y^{2}\right\},
$$


which uses 849 cosets and is the canonic variant of the Table 1 presentation. Setting $x=a b$ and $y=a B$ in the second relator of this presentation to produce a relator $w_{3}$ gives

$$
\left\{a, b \mid a^{2}, b^{3}, w_{3}\right\}
$$

as an explicit presentation for $L_{2}(8)$ as a one-relator quotient of $C_{2} * C_{3}$ where $w_{3}$ has length 26 , which improves on the shortest extra relator with length 30 found for one specific generating set in [15]. If we count only relator length, then shorter efficient presentations exist: for example, on three generators and with length 16 ,

$$
\left\{a, b, c \mid a^{2} b A c, a b c A B, b^{2} C B C C\right\}
$$

which requires 552 cosets.

Note that various deficiency-one presentations, including

$$
\begin{aligned}
& \left\{a, b \mid(a b)^{2}, a^{7}, a^{3} b A b^{3} A b\right\}, \\
& \left\{a, b \mid a^{2}, b^{7}, a b a B a b^{3} a b^{3} a B\right\} \\
& \left\{a, b \mid a^{2}, b^{9}, a b a b^{4} a b a B a B\right\},
\end{aligned}
$$

allow enumeration of the cosets of the trivial group using a total of 504 cosets - that is, without the definition of any redundant cosets.

\section{3. $A_{7}$}

For $A_{7}$, we investigated 505 distinct generating pairs and found 330 three-relator presentations for preimages of $A_{7}$ that define finite perfect groups. Amongst these were many efficient presentations for $A_{7}$ itself, and also one-relator quotients of $C_{2} * C_{7}, C_{3} * C_{4}$, $C_{3} * C_{5}$ and $C_{3} * C_{7}$, which presented $A_{7}$ or a stem extension. We can use Theorem 4.1 to construct an efficient presentation for $\widehat{A}_{7}$ from any of these one-relator quotients of $C_{l} * C_{m}$.

The tabulated presentation for $A_{7}$ was obtained from a census of short two-generator, three-relator presentations. For $\widehat{A}_{7}$, we tabulate a presentation from a census of short tworelator presentations for perfect groups, which is the unique shortest canonic two-relator presentation for this group. A 2-longer canonic presentation enumerates better:

$$
\left\{a, b \mid a^{3} b A b A b, a^{2} B a b A B^{2} A b^{2} A B\right\}
$$

uses 21125 cosets.

\section{4. $L_{2}(16)$}

For $L_{2}(16)$, we investigated 524 distinct generating pairs and, in contrast to the situation with $L_{2}(8)$, we found fifteen efficient presentations directly. We also found one-relator quotients of $C_{2} * C_{3}, C_{2} * C_{5}$ and $C_{2} * C_{15}$ that present the group. Theorem 4.1 enables us to construct an efficient presentation for $L_{2}(16)$ from any of these. We tabulate a length-18 presentation from a census of short two-relator presentations for perfect groups, which is the unique shortest canonic two-relator presentation for this group. A canonic length-19 presentation,

$$
\left\{a, b \mid a^{2} b A B A b, a b^{2} A b^{2} a B^{5}\right\},
$$

enumerates much better, using 4575 cosets. The group arises implicitly as a one-relator quotient of $C_{2} * C_{3}$ via the length-23 presentation:

$$
\left\{x, y \mid x y x Y X Y, x^{3} Y x Y^{4} x Y x^{3} Y^{3}\right\},
$$

which uses 7515 cosets, and is the canonic variant of the Table 1 presentation. 


\section{5. $L_{3}(3)$}

For $L_{3}(3)$, we investigated 1275 distinct generating pairs, and found twelve efficient presentations directly. We also found one-relator quotients of $C_{2} * C_{3}, C_{3} * C_{4}$ and $C_{3} * C_{8}$ that present the group. Theorem 4.1 enables us to construct an efficient presentation for $L_{3}(3)$ from any of these. We tabulate a length-21 presentation from a census of short tworelator presentations for perfect groups. Three-generator efficient presentations with length 20 exist;

$$
\left\{a, b, c \mid a^{2} b A b, a b c b C a C, a c A c b^{3} C\right\}
$$

is the best canonic one found for total cosets, 92576 . The best canonic two-generator presentation for coset enumeration that we found has length 24 ,

$$
\left\{a, b \mid a^{4} b A b^{2} A B^{2}, a^{4} B^{2} a b^{5}\right\},
$$

and uses 27778 cosets.

\section{6. $U_{3}(3)$}

This group has been considered in detail by Havas, Newman and O'Brien [19] in the context of efficient semigroup presentations. For $U_{3}(3)$, we investigated 1442 distinct generating pairs, and found two efficient presentations amongst presentations on these generating sets. We also found one-relator quotients of $C_{3} * C_{4}, C_{3} * C_{7}$ and $C_{3} * C_{8}$ that present the group. Theorem 4.1 enables us to construct an efficient presentation for $U_{3}(3)$ from any of these. We tabulate a presentation from a census of short two-relator presentations for perfect groups, the unique canonic presentation with length 19 . This may be obtained by amalgamating the power relations in a one-relator quotient of $C_{3} * C_{4}$. A canonic presentation with length 20 ,

$$
\left\{a, b \mid a^{2} b A^{2} b^{2} A B, a^{3} b^{2} A B^{2} A^{2} B\right\},
$$

enumerates better, using 15583 cosets. A three-generator, three-relator presentation with length 19,

$$
\left\{a, b, c \mid a^{2} b A b, a b c B c A C, a B^{2} C b A C\right\},
$$

uses fewer cosets: 10673. A canonic presentation with length 21,

$$
\left\{a, b \mid a^{2} b a^{2} B^{2}, a^{3} b A B A B A b a^{3} B\right\},
$$

is better again, at 8878 cosets.

\section{7. $L_{2}(25)$}

As the number of generating sets goes up, it becomes very time-consuming to look at all three-relator subsets for each generating set. $L_{2}(25)$ has 1016 distinct generating pairs. We investigated only a sample of three-relator subsets, and found many efficient presentations for $L_{2}(25)$, including one-relator quotients of $C_{2} * C_{3}, C_{2} * C_{5}$ and $C_{2} * C_{13}$. We can use Theorem 4.1 to construct an efficient presentation for $\widehat{L}_{2}(25)$ from any of these onerelator quotients of $C_{l} * C_{m}$. For $L_{2}(25)$, we tabulate a presentation from a census of short two-generator, three-relator presentations. For its cover, we tabulate a presentation from a census of short two-relator presentations for perfect groups; this is the unique shortest canonic two-relator presentation for this group. 


\section{8. $M_{11}$}

For $M_{11}$, we investigated 3297 distinct generating pairs, and found many efficient presentations directly, including two with what turns out to be minimal length. The group also arises readily as one-relator quotients of $C_{2} * C_{5}, C_{4} * C_{5}, C_{3} * C_{8}, C_{5} * C_{6}$ and $C_{5} * C_{8}$.

The census of short two-relator presentations for perfect groups reveals exactly one canonic two-relator presentation for the group with length 19, and nothing shorter. This presentation must be shortest, by an extension of the argument in [18]; Kenne [20] had already found a form of it.

However, we tabulate one that enumerates better. In this case, since there is only one canonic presentation of this length, we find the best coset enumeration for a shortest presentation using the method of [17] applied to all different two-generator, length-19 presentations for the group.

\section{9. $L_{2}(27)$}

$L_{2}(27)$ has 864 distinct generating pairs. We investigated only a sample of three-relator subsets, and found many efficient presentations. We also found one-relator quotients of $C_{2} * C_{3}, C_{2} * C_{7}$ and $C_{3} * C_{7}$. We can use Theorem 4.1 to construct an efficient presentation for $\widehat{L}_{2}$ (27) from any of these one-relator quotients of $C_{l} * C_{m}$. For $L_{2}(27)$, we tabulate a presentation from a census of short two-generator, three-relator presentations. For its cover, we tabulate a presentation from a census of short two-relator presentations for perfect groups. Notice the enormous improvement in coset-enumeration performance of our presentation for $\widehat{L}_{2}(27)$, compared with the Table 1 presentation.

\subsection{0. $A_{8}$}

For $A_{8}$, we investigated 3868 distinct generating pairs. We found a number of efficient presentations for $A_{8}$, including some, like the one tabulated (from a census of short two-generator, three-relator presentations), that are one-relator quotients of $C_{2} * C_{7}$. This implicitly solves the first of the previously unsolved problems by providing a base for constructing an efficient presentation for $\widehat{A}_{8}$ via Theorem 4.1 and Corollary 4.2. A longer presentation for $A_{8}$ (from the distinct generating pairs),

$$
\left\{a, b \mid B^{7}, b a b A^{3} B a^{2} b^{2}, B^{2} A b A^{2} b^{2} a^{2} B\right\},
$$

uses only 22363 cosets. Presentations for $A_{8}$ also arise as one-relator quotients of $C_{4} * C_{7}$. For $\widehat{A}_{8}$, we tabulate a presentation from a census of short two-relator presentations for perfect groups. This presentation can also be obtained by simply amalgamating the power relators in a presentation for $A_{8}$ as a one-relator quotient of $C_{2} * C_{7}$, namely

$$
\left\{a, b \mid\left(A^{2} b\right)^{2}, a^{7}, a^{2} b^{2} A B a b^{2} A B a B\right\} .
$$

The best coset enumeration that we have found for a canonic, two-generator, efficient presentation for $\widehat{A}_{8}$ uses 63560 cosets:

$$
\left\{a, b \mid a^{2} b A b a B A^{2} B^{2}, a^{2} b A b A b a b A b a b\right\} .
$$

If we count only relator length, then shorter efficient presentations exist: for example, on three generators and with length 19 ,

$$
\left\{a, b, c \mid a^{2} b A b, b c b c B C, a b c A b c A c\right\},
$$

which requires 52934 cosets. 


\subsection{1. $L_{3}(4)$}

For $L_{3}(4)$, we investigated 779 distinct generating pairs. This is a moderate enough number. However, a problem with this group is that it is the smallest simple group with multiplier of rank 2. This implies that efficient presentations have four relations, and there are very many four-relator subsets of the presentations on the distinct generating pairs. We investigated all of them, and found many efficient presentations. In the same way that $C_{l} * C_{m}$ is a good basis for three-relator presentations, the group $(l, m, n)=\left\{a, b \mid a^{l}, b^{m},(a b)^{n}\right\}$ is a good basis for four-relator presentations. Included in the efficient presentations of $L_{3}(4)$ that we found were a substantial number of variants of one-relator quotients of $(5,5,3)$, but no one-relator quotients of any other $(l, m, n)$. Having observed this, we listed one-relator quotients of $(5,5,3)$, and found four canonic extra relators with length 13 that yield $L_{3}(4)$, and we list the one with best coset-enumeration behaviour.

Unfortunately, none of the efficient presentations for $L_{3}(4)$ that were found this way initially enabled us to construct an efficient presentation for $\widehat{L}_{3}(4)$ by amalgamating relators. So we looked at three-relator preimages of $L_{3}(4)$, hoping to find a stem extension that we could use as a starting-point. A presentation satisfying Theorem 4.1 would have been ideal. We found no such presentation; however, we did find eleven variants of

$$
\left\{a, b \mid a^{5},(A B A b)^{3}, w_{3}\right\}
$$

that present a stem extension, 12. $L_{3}(4)$. When we simply amalgamate the power relators, we obtain as a first efficient presentation for $\widehat{L}_{3}(4)$ :

$$
\left\{a, b \mid a^{5}(A B A b)^{3}, a^{2} B^{2} A^{2} b a B A b\right\} .
$$

This presentation is difficult to use for coset enumeration, using 145807531 cosets. This practical construction is an instance of the following easy theorem, which can in fact be viewed as a precursor to Theorem 4.1 and Corollary 4.2.

TheORem 5.1. Let $G$ be a finite simple group. Suppose that $G$, or some stem extension of $G$, can be presented as

$$
\left\{a, b \mid u(a, b)^{p}=v(a, b)^{q}=w(a, b)=1\right\} .
$$

In addition, suppose that $\widetilde{G}$, presented by

$$
\left\{a, b \mid u(a, b)^{k p} v(a, b)^{l q}=w(a, b)=1\right\},
$$

is perfect, and is generated by $u(a, b)$ and $v(a, b)$. Then $\widetilde{G}$ is the covering group of $G$.

The structure of our first efficient presentation for $\widehat{L}_{3}(4)$ suggests that it might be profitable to study one-relator quotients of $C_{3} * C_{5}$ more carefully. We did so, and uncovered various useful presentations that we had ignored in our censuses because the coset enumerations attempted during the census process failed due to space limitations. These include as presentations for 12. $L_{3}(4)$ :

$$
\begin{aligned}
& P_{1}=\left\{a, b \mid a^{3}, b^{5}, a b a b A b A B^{2} A b^{2} a b^{2} a B\right\} ; \\
& P_{2}=\left\{a, b \mid a^{3}, b^{5}, a b^{3} a B^{2} A B^{2} A B A b a b a b^{2}\right\} .
\end{aligned}
$$

Over the trivial subgroup, ACE uses 41128739 and 60689170 cosets, respectively. Theorem 4.1, applied to $P_{1}$, yields a variant of $P_{2}$. The power relations of $P_{2}$ amalgamate simply to give

$$
\left\{a, b \mid a^{3} b^{5}, a b^{3} a B^{2} A B^{2} A B A b a b a b^{2}\right\}
$$


as an efficient presentation for $\widehat{L}_{3}(4)$. This presentation uses a total of 315894198 cosets over the trivial subgroup, worse than our first presentation.

Finally, by looking carefully at one-relator quotients of $\left\{a, b \mid a^{5},(a b)^{3}\right\}$, we discovered that

$$
\left\{a, b \mid a^{5},(a b)^{3}, a^{2} b A^{2} b A b^{2} A B^{2} a B\right\}
$$

is a presentation for $12 . L_{3}(4)$, leading to

$$
\left\{a, b \mid A^{5}(a b)^{3}, a^{2} b A^{2} b A b^{2} A B^{2} a B\right\}
$$

as a presentation for $\widehat{L}_{3}(4)$. This presentation has length 23 , and its canonic version is in our tabulation, using a more modest 30181644 cosets over the trivial subgroup. In retrospect, we see that the difficulty of the coset enumeration meant that the presentation was initially classified as an overflow in our census process.

\subsection{2. $S_{4}(3)$}

For $S_{4}(3)$, we investigated 5993 distinct generating pairs. Presentations on these include ten that are already efficient, and 105 with four relators. From the 105 deficiency-two presentations, we can build another sixty-seven efficient presentations. Seven of these efficient presentations include relators $u(a, b)^{4}$ and $v(a, b)^{5}$, where $u(a, b)$ and $v(a, b)$ generate the free group on $a$ and $b$. Using these and Corollary 4.2, we obtained efficient presentations for $\widehat{S}_{4}(3)$, for which efficient presentations had not previously been known. For $S_{4}(3)$, we tabulate a presentation from a census of short two-generator, three-relator presentations. For its cover, we tabulate a presentation from a census of short two-relator presentations for perfect groups. Adding the relator $a^{5}$ to the presentation for $\widehat{S}_{4}(3)$ and simplifying gives a presentation for $S_{4}(3)$ as a one-relator quotient of $C_{4} * C_{5}$ that has the same length as the tabulated presentation but enumerates somewhat worse, at 44575 cosets.

\subsection{3. $\mathrm{Sz}(8)$}

For $\mathrm{Sz}(8)$, we investigated 4577 distinct generating pairs. This is the second smallest simple group with multiplier of rank 2 . We found very many efficient presentations but, in contrast to the situation with $L_{3}(4)$, none as variants of one-relator quotients of $(l, m, n)$. However, they did arise as two-relator quotients of $C_{5} * C_{7}$ and $C_{7} * C_{7}$. We tabulate a two-relator quotient of $C_{5} * C_{7}$, and mention that a two-relator quotient of $C_{7} * C_{7}$,

$$
\left\{a, b \mid A^{7}, B^{7}, B a B a B A b^{2} A B, a^{2} b A^{3} B a b A B\right\},
$$

has the same length and enumerates almost as well (29859 cosets). There are very many efficient presentations for this group that enumerate well.

By simply looking at two-relator preimages of the presentations on the distinct generating pairs, we find many efficient presentations for $\widehat{\mathrm{Sz}}(8)$. We tabulate one found in a census of short two-relator presentations for perfect groups. A three-generator, threerelator presentation with length 18 ,

$$
\left\{a, b, c \mid a^{2} b c B, a b a C A c, a^{2} B C A b c\right\},
$$

uses fewer cosets, 131283.

\subsection{4. $L_{2}(32)$}

For $L_{2}(32)$, we investigated 3351 distinct generating pairs and (not unlike the situation with $L_{2}(16)$ ) we found many efficient presentations directly. We also found one-relator 
quotients of $C_{2} * C_{3}$ and $C_{2} * C_{11}$ that present the group. We tabulate a length-21 presentation from a census of short two-relator presentations for perfect groups.

\subsection{5. $L_{2}(49)$}

For $L_{2}(49)$, we investigated 7553 distinct generating pairs, and we found many efficient presentations directly. We also found one-relator quotients of $C_{2} * C_{3}, C_{2} * C_{5}, C_{3} * C_{4}$ and $C_{4} * C_{5}$ that present the group. We can use Theorem 4.1 to construct an efficient presentation for $\widehat{L}_{2}$ (49) from any of these one-relator quotients of $C_{l} * C_{m}$. For $L_{2}(49)$, we tabulate a presentation from a census of short two-generator, three-relator presentations. For its cover, we tabulate a presentation obtained from looking at presentations on distinct generating pairs. Its canonic version,

$$
\left\{a, b \mid a^{2} b a^{2} b A^{2} b, a b^{2} A b^{2} a B^{3}\right\},
$$

is the unique shortest canonic two-relator presentation for this group, but enumerates a little worse, at 265430 cosets.

Other longer presentations enumerate better. For $L_{2}(49)$,

$$
P=\left\{x, y \mid\left(x Y^{2}\right)^{2},(x Y)^{3}, x y^{2}\left(x^{2} y^{3} x^{2}\right)^{2} y^{2}\right\}
$$

uses 59769 cosets. For its cover, the Table 1 presentation (which is based on the same long relator as in $P$ ) is better; the presentation

$$
\left\{a, b \mid a^{3} b A^{2} B A^{2} b, a^{4} B a B^{3} a B\right\}
$$

is better again, using 126569 cosets. A three-generator, three-relator presentation with length 17 ,

$$
\left\{a, b, c \mid a^{2} b A b, a c A C C, b^{3} c B^{2} c\right\}
$$

uses 432168 cosets.

\subsection{6. $U_{3}(4)$}

For $U_{3}(4)$, we investigated 7778 distinct generating pairs. In a partial search of tworelator preimages on these generating sets, we detected only proper preimages. However, among the three-relator preimages we found a number of one-relator quotients of $C_{3} * C_{5}$ that present the group. We can use Theorem 4.1 to construct an efficient presentation from any of them. We tabulate, from a census of short two-relator presentations for perfect groups, the length-22 canonic presentation that enumerates best.

A length-23 canonic presentation enumerates substantially better:

$$
\left\{a, b \mid a^{3} b A^{2} B A^{2} b, a^{3} b^{2} a^{2} B^{3} A B^{2}\right\}
$$

uses 416708 cosets. We found a one-relator quotient of $C_{2} * C_{3}$ :

$$
\left\{a, b \mid(b A)^{3},(b A b)^{2}, a^{7} b^{2} a^{4} b a b^{2} a^{2} b^{2}\right\}
$$

which requires 98318 cosets. Applying Corollary 4.2 and simplifying, we obtain

$$
\left\{r, s \mid s^{3}, r^{2},(s r)^{7}(S r)^{2}(s r)^{4} \operatorname{Sr} s r(S r)^{2}(s r)^{2}(S r)^{2}\right\} .
$$

Then, applying Theorem 4.1, we can obtain as an example efficient presentation

$$
\left\{r, s \mid s^{3} r^{2},(s R)^{7}(S r)^{2}(s r)^{4} \operatorname{Sr} s R(S r)^{2}(s r)^{2}(S r)^{2}\right\},
$$

which uses 477214 cosets. 


\subsection{7. $M_{12}$}

$M_{12}$ is the largest simple group in our catalogue. It has 19801 distinct generating pairs, and $\widehat{M}_{12}$ has 77979 distinct generating pairs. We investigated all the distinct generating sets for $M_{12}$, and found many efficient presentations for the group. Unfortunately, none was of a form suitable to apply Theorem 4.1 or Theorem 5.1. We tabulate the shortest instance found with best total cosets. The length-39 presentation

$$
\left\{a, b \mid b A B a B A B a^{2} b^{2} a B^{2} a b, A B a^{3} B A^{2}, a B a B a B A^{2} b^{2} A b^{2} a b\right\}
$$

enumerates quite well, using 106282 cosets.

We investigated some of the distinct generating sets for the cover, and failed to find any efficient presentations, or any presentations to which Theorem 4.1 or Theorem 5.1 could be applied.

The group $\widehat{M}_{12}$ is not only the last group in our catalogue, but was the last for which we were able to find an efficient presentation. It did not arise in our censuses. In the end, it succumbed to our third method; we studied longer, one-relator quotients of $C_{2} * C_{3}$. On mapping $C_{2} * C_{3}$ onto a representation of $M_{12}$ satisfying presentation 13.1 of [9], we found various words with length from 54 up which mapped onto the identity. Furthermore, in many cases we could show that the words sufficed to produce $\widehat{M}_{12}$ as a one-relator quotient of $C_{2} * C_{3}$.

Starting with $\left\{a, b \mid a^{2}, b^{3}\right\}$ and defining $x=a b$ and $y=a B$, we found, inter alia, that the word

$$
w=x^{2} y^{3} x^{2} y x y x^{2} y^{2} x y x y^{5} x^{5}
$$

maps to the identity in the representation of $M_{12}$. We investigated the group presented by

$$
\left\{a, b \mid a^{2}, b^{3}, w\right\}
$$

and found (by coset enumeration) that it is $\widehat{M}_{12}$. Theorem 4.1 then delivers us many efficient presentations for $\widehat{M}_{12}$, including:

$$
\left\{a, b \mid a^{2} b^{3},(A b)^{2}(a B)^{3}(a b)^{2}(a B)(a b)(a B)(a b)^{2}(a B)^{2}(a b)(a B)(A b)(A B)^{5}(A b)^{5}\right\},
$$

which was chosen somewhat arbitrarily from among the options. We can enumerate the cosets of the trivial subgroup for this presentation using a total of 27890300 cosets. From this, we constructed the shorter presentation that we tabulate.

\section{Review}

In retrospect, we observe that many deficiency-zero presentations that we list can be viewed as results of applications of Theorem 4.1, Corollary 4.2 or Theorem 5.1. These include most of the deficiency-zero presentations in both Table 1 and Table 2.

Observe that in our Table 2 presentations, all enumerations over the trivial subgroup can be completed using a total of less than 31 million cosets. This is in sharp contrast to many earlier efficient presentations, for which the coset enumerations are much harder. Perhaps the worst example is the first published presentation for $L_{2}(49)$, referred to in [8] and appearing in [6], which is theoretically correct but fails to complete enumerations over cyclic subgroups even when allowed to define more than $2 \times 10^{9}$ cosets.

It suffices to say that our methods enable us to produce efficient presentations that are both short and computationally useful. 


\section{Open problems}

We list below some standard open problems, and some that have arisen during the work described in this paper.

(1) Is every simple group efficient? If not, which is the smallest inefficient simple group? Only one simple group with order less than one million is a candidate, $S_{4}(4)$. In particular, is $L_{2}\left(2^{n}\right)$ efficient for all $n$ ? Note that this has a positive solution for $n=2,3,4,5,6$.

(2) Does the covering group of every finite simple group have a balanced presentation?

(3) Is $A_{n}$ efficient for all $n$ ? This has a positive solution for $n \leqslant 9$. A much weaker question even appears to be open: Is there a two-generator presentation for $A_{n}$ with $k$ relators, where $k$ is independent of $n$ ?

(4) Is there a group with two generators that has an efficient presentation on three or more generators, but not on two generators?

(5) Do there exist groups with efficient presentations on one generating set but not on another? In particular, do the small simple groups have efficient presentations on every generating pair?

\section{References}

1. Abd Ghafur bin Ahmad, 'The unsolvability of efficiency for groups', Southeast Asian Math. Bull. 22 (1998) 331-336. 266

2. Wieb Bosma, John Cannon and Catherine Playoust, 'The Magma algebra system, I: the user language', J. Symbolic Comput. 24 (1997) 235-265; see also http://magma.maths.usyd.edu.au/magma/ 267

3. C. M. Campbell and E. F. Robertson, 'A deficiency zero presentation for $\operatorname{SL}(2, p)$ ', Bull. London Math. Soc. 12 (1980) 17-20. 267

4. C. M. Campbell, E. F. Robertson, T. Kawamata, I. Miyamoto and P. D. Williams, 'Deficiency zero presentations for certain perfect groups', Proc. Roy. Soc. Edinburgh 103A (1986) 63-71. 269, 270

5. Colin M. CAmpbell and Edmund F. Robertson, 'The efficiency of simple groups of order $<10^{5}$, Comm. Algebra 10 (1982) 217-225. 267, 269

6. Colin M. CAmpbell and Edmund F. Robertson, 'On a class of groups related to SL $\left(2,2^{n}\right)$ ', Computational group theory (ed. Michael D. Atkinson, Academic Press, London, 1984) 43-49. 280

7. Colin M. Campbell, George Havas, Alexander Hulpke and Edmund F. Robertson, 'Efficient simple groups', Comm. Algebra 31 (2003) 5191-5197. 266, 268

8. Colin M. Campbell, E. F. Robertson and P. D. Williams, 'Efficient presentations for finite simple groups and related groups', Groups - Korea 1988, Lecture Notes in Math. 1398 (ed. A. C. Kim and B. H. Neumann, Springer, New York, 1989) 65-72. 266,280 
9. John J. Cannon, John McKay and Kiang Chuen Young, 'The non-abelian simple groups $G,|G|<10^{5}$ — presentations', Comm. Algebra 7 (1979) 1397-1406. 280

10. J. H. Conway, R. T. Curtis, S. P. Norton, R. A. Parker and R. A. Wilson, Atlas of finite groups (Oxford Univ. Press, Oxford, 1985). 266

11. H. S. M. Coxeter, 'The abstract groups $G^{m, n, p}$ ', Trans. Amer. Math. Soc. 45 (1939) 73-150. 273

12. Dragomir Ž. ĐoKović, 'Presentations of some finite simple groups', J. Austral. Math. Soc. Ser. A 45 (1988) 143-168. 271

13. Greg Gamble, Alexander Hulpke, George Havas and Colin Ramsay, GaP package ACE; Advanced Coset Enumerator, 2004;

http://www.gap-system.org/Packages/ace.html. 268

14. The GAP Group, GAP - Groups, algorithms, and programming, Version 4.3, 2000; http://www.gap-system.org/. 267

15. ThE GAP GROUP, Balanced presentations for covering groups of simple groups, 2004; http://www.gap-system.org/Doc/Examples/balanced.html. 268, 274

16. George Havas and Colin Ramsay, 'Proving a group trivial made easy: a case study in coset enumeration', Bull. Austral. Math. Soc. 62 (2000) 105-118. 268

17. George Havas and Colin Ramsay, Coset enumeration: ACE version 3.001, 2001; http://www.itee.uq.edu.au/ havas/ace3001.tar.gz. 267, 276

18. George Havas and Colin Ramsay, 'Short balanced presentations of perfect groups', Groups St Andrews 2001 in Oxford, vol. 1, London Math. Soc. Lecture Note Ser. 304 (ed. C. M. Campbell, E. F. Robertson and G. C. Smith, Cambridge Univ. Press, Cambridge, 2003) 238-243. 266, 268, 271, 273, 276

19. George Havas, M. F. Newman and E. A. O'Brien, 'On the efficiency of some finite groups’, Comm. Algebra 32 (2004) 649-656. 267, 269, 272, 275

20. P. E. Kenne, 'Efficient presentations for three simple groups', Comm. Algebra 14 (1986) 797-800. 269, 276

21. The New York Group Theory Cooperative, Magnus;

http://www.grouptheory.org/. 267

22. Edmund F. Robertson, 'Efficiency of finite simple groups and their covering groups', Contemp. Math. 45 (1985) 287-294. 269

23. J. G. Sunday, 'Presentations of the groups $\operatorname{SL}(2, m)$ and PSL(2, $m)$ ', Canad. J. Math. 24 (1972) 1129-1131. 267

24. J. WiEgold, 'The Schur multiplier: an elementary approach', Groups - St Andrews 1981, London Math. Soc. Lecture Note Ser. 71 (ed. C. M. Campbell and E. F. Robertson, Cambridge Univ. Press, Cambridge, 1982) 137-154. 266 
Colin M. Campbell colincemcs.st-and.ac.uk

http://www-groups.mcs.st-andrews.ac.uk/ colinc/

School of Mathematics and Statistics

University of St Andrews

North Haugh, St Andrews

Fife KY16 9SS

United Kingdom

George Havas havaseitee.uq.edu.au

http://www.itee.uq.edu.au/ havas/

ARC Centre for Complex Systems

School of Information Technology and Electrical Engineering

The University of Queensland

Queensland 4072

Australia

Colin Ramsay crameitee.uq.edu.au

http: //www.itee.uq.edu.au/ cram/

ARC Centre for Complex Systems

School of Information Technology and Electrical Engineering

The University of Queensland

Queensland 4072

Australia

Edmund F. Robertson edmundemcs.st-and.ac.uk

http://www-groups.mcs.st-andrews.ac.uk/ redmund/

School of Mathematics and Statistics

University of St Andrews

North Haugh, St Andrews

Fife KY16 9SS

United Kingdom 